\title{
LIMITED-MEMORY REDUCED-HESSIAN METHODS FOR LARGE-SCALE UNCONSTRAINED OPTIMIZATION
}

\author{
PHILIP E. GILL* AND MICHAEL W. LEONARD ${ }^{\dagger}$
}

\begin{abstract}
Limited-memory BFGS quasi-Newton methods approximate the Hessian matrix of second derivatives by the sum of a diagonal matrix and a fixed number of rank-one matrices. These methods are particularly effective for large problems in which the approximate Hessian cannot be stored explicitly.

It can be shown that the conventional BFGS method accumulates approximate curvature in a sequence of expanding subspaces. This allows an approximate Hessian to be represented using a smaller reduced matrix that increases in dimension at each iteration. When the number of variables is large, this feature may be used to define limited-memory reduced-Hessian methods in which the dimension of the reduced Hessian is limited to save storage. Limited-memory reduced-Hessian methods have the benefit of requiring half the storage of conventional limited-memory methods.

In this paper, we propose a particular reduced-Hessian method with substantial computational advantages compared to previous reduced-Hessian methods. Numerical results from a set of unconstrained problems in the CUTE test collection indicate that our implementation is competitive with the limited-memory codes L-BFGS and L-BFGS-B.
\end{abstract}

Key words. Unconstrained optimization, quasi-Newton methods, BFGS method, reducedHessian methods, conjugate-direction methods

AMS subject classifications. 65K05, 90C30

1. Introduction. BFGS quasi-Newton methods have proved reliable and efficient for the unconstrained minimization of a smooth nonlinear function $f: \mathbb{R}^{n} \rightarrow \mathbb{R}$. However, the need to store an $n \times n$ approximate Hessian has limited their application to problems with a small-to-moderate number of variables (say, less than 500). For larger $n$ it is necessary to use methods that do not require the storage of a full $n \times n$ matrix. Sparse quasi-Newton updates can be applied if the Hessian has a significant number of zero entries (see, e.g., Powell and Toint [29], Fletcher [10]). However, if the Hessian is dense, as is often the case for certain subproblems arising in nonlinearly constrained optimization, other methods must be used. Such methods include conjugate-gradient methods, limited-memory quasi-Newton methods, and limited-memory reduced-Hessian quasi-Newton methods.

Conjugate-gradient methods require storage for only a few $n$-vectors (see, e.g., Gill, Murray, and Wright [17, pp. 144-150]). These methods can be equivalent to the BFGS quasi-Newton method on a quadratic function, but they are generally acknowledged to be less robust on general nonlinear problems (see Gill and Murray [14] for some numerical comparisons). Limited-memory quasi-Newton methods also require storage of few $n$-vectors but have a more explicit relationship with quasiNewton methods. Limited-memory methods exploit the fact that the approximate Hessian (or its inverse) can be written as the sum of a diagonal matrix and a number of rank-one matrices. This allows the search direction to be calculated as a simple linear combination of the vectors that define each rank-one update. The idea of

*Department of Mathematics, University of California, San Diego, La Jolla, California 920930112, USA (pgill@ucsd.edu). Research supported by the National Science Foundation grants DMI9424639, CCR-9896198, and DMS-9973276 and Office of Naval Research grant N00014-96-1-0274.

$\dagger^{\dagger}$ Department of Mathematics, University of California, San Diego, La Jolla, California 920930112, USA (mleonard@na-net.ornl.gov). Research supported by National Science Foundation grant DMI-9424639. 
a limited-memory method is to store a fixed number $m(m \ll n)$ of pairs of update vectors and to discard older pairs as new ones are computed. These methods appeared in the early 1980s (see, e.g., Shanno [30] and Nocedal [26]), and they have now been developed to a considerable level of sophistication (see Byrd, Nocedal, and Schnabel [5] and Kaufman [19]). Limited-memory approximate Hessians may be used directly in a conventional quasi-Newton method, or they may be used as preconditioners for a nonlinear conjugate-gradient method (see, e.g., Buckley [2,3], Gill, Murray, and Wright [17, pp. 151-152], Morales and Nocedal [22], and Nazareth [25]).

A different approach has been taken by Fenelon [8] and Siegel [33], who independently proposed methods in which the curvature is accumulated in a subspace spanned by a set of $m$ independent vectors. These reduced-Hessian methods exploit the fact that quasi-Newton methods accumulate approximate curvature in a sequence of expanding subspaces (see Gill and Leonard [13]). Reduced-Hessian methods represent the approximate Hessian using a smaller reduced matrix that increases in dimension at each iteration. This reduced matrix incorporates curvature information that has been accumulated during earlier iterations and allows the search direction to be calculated from a linear system that is smaller than that used in conventional methods.

In this paper we propose the limited-memory method L-RHR, which may be viewed as a limited-memory variant of the reduced-Hessian method RHR of Gill and Leonard [13]. L-RHR has two features in common with the limited-memory method of Siegel [33]: a basis of search directions is maintained for the sequence of $m$-dimensional subspaces, and an implicit orthogonal decomposition is used to define an orthonormal basis for each subspace. However, L-RHR is different from Seigel's algorithm in several ways: (i) L-RHR updates the Cholesky factor of the reduced Hessian instead of updating an explicit reduced inverse Hessian; (ii) the formulation of L-RHR as a modification of RHR allows the application of Hessian reinitialization, which is shown to greatly enhance performance on large problems; and (iii) L-RHR employs selective basis reorthogonalization to improve robustness for moderate values of the subspace dimension. Property (i) implies that in exact arithmetic, even if implemented without Hessian reinitialization and selective reorthogonalization, the L-RHR iterates are different from those of Siegel's method (see section 3.6). Properties (i)-(iii) not only provide substantial improvements in efficiency compared to Siegel's method, but also make reduced-Hessian methods competitive with the state-of-the-art limited-memory method L-BFGS-B of Zhu et al. [34]. L-RHR requires the storage of an $n \times m$ matrix, two $m \times m$ nonsingular upper-triangular matrices, and a fixed number of $n$ - and $m$ vectors. For a given $m$, this is approximately half the storage required for L-BFGS-B to represent essentially the same amount of second-derivative information. Moreover, L-RHR requires fewer floating-point operations per iteration, which results in smaller overall computation times on many problems.

The paper is organized as follows. In section 2 we briefly review various theoretical aspects of reduced-Hessian quasi-Newton methods, including the definition of Algorithm RHR, a reduced-Hessian method with Hessian reinitialization. Algorithm RHR provides the theoretical framework for the limited-memory algorithm L-RHR proposed in section 3. We give algorithms for maintaining both gradient- and search-direction subspace bases, and it is shown that L-RHR has the property of finite termination on a strictly convex quadratic function. To simplify the discussion, the algorithms of sections 2-3 are stated with the assumption that all computations are performed in exact arithmetic. The effects of rounding error and the use of reorthogonalization are discussed in sections 4.1, 4.2, and 4.3. Finally, section 5 includes some numerical re- 
sults obtained when various limited-memory reduced-Hessian algorithms are applied to test problems from the CUTE test collection of Bongartz et al. [1]. It is shown that reinitialization and selective reorthogonalization (in conjunction with an explicit factorization for the subspace basis) give, respectively, significantly fewer function evaluations and increased robustness compared to Siegel's method. Section 5 also includes comparisons of L-RHR with two alternative implementations of the conventional limited-memory BFGS method.

Unless explicitly indicated otherwise, $\|\cdot\|$ denotes the vector two-norm or its subordinate matrix norm.

2. Motivation. The BFGS method generates a sequence of iterates $\left\{x_{k}\right\}$ such that $x_{k+1}=x_{k}+\alpha_{k} p_{k}$, where $p_{k}$ is the search direction and $\alpha_{k}$ is a scalar step length. The search direction satisfies $H_{k} p_{k}=-\nabla f\left(x_{k}\right)$, where $H_{k}$ is an approximate Hessian. The application of the BFGS update to $H_{k}$ gives a matrix $H_{k}^{\prime}$ such that

$$
H_{k}^{\prime}=H_{k}-\frac{1}{\delta_{k}^{T} H_{k} \delta_{k}} H_{k} \delta_{k} \delta_{k}^{T} H_{k}+\frac{1}{\gamma_{k}^{T} \delta_{k}} \gamma_{k} \gamma_{k}^{T},
$$

where $\delta_{k}=x_{k+1}-x_{k}, g_{k}=\nabla f\left(x_{k}\right)$, and $\gamma_{k}=g_{k+1}-g_{k}$. A conventional BFGS method then defines $H_{k+1}=H_{k}^{\prime}$ (another choice for $H_{k+1}$ is discussed in section 2.2). If $H_{0}$ is symmetric and positive definite, and if $\alpha_{k}$ is such that the approximate curvature $\gamma_{k}^{T} \delta_{k}$ is positive, then $H_{k}$ is symmetric positive definite for all $k \geq 0$. Conditions imposed on the step length by practical step-length algorithms can ensure both positivity of the approximate curvature and sufficient descent. This is the case, for example, for any $\alpha_{k}$ satisfying the Wolfe conditions

$$
f\left(x_{k}+\alpha_{k} p_{k}\right) \leq f\left(x_{k}\right)+\mu \alpha_{k} g_{k}^{T} p_{k} \quad \text { and } \quad g_{k+1}^{T} p_{k} \geq \eta g_{k}^{T} p_{k},
$$

where the constants $\mu$ and $\eta$ are chosen so that $0 \leq \mu<\eta<1$ and $\mu<\frac{1}{2}$.

The need to solve a linear system for $p_{k}$ makes it convenient to use the uppertriangular Cholesky factor $C_{k}$ such that $H_{k}=C_{k}^{T} C_{k}$. In this case, the Cholesky factor $C_{k+1}$ of $H_{k+1}$ is obtained from a rank-one change to $C_{k}$ (see Dennis and Schnabel [7]). We omit the details of this procedure and simply write $C_{k+1}=$ update $\left(C_{k}, \delta_{k}, \gamma_{k}\right)$.

2.1. Reduced-Hessian methods. Reduced-Hessian methods provide an alternative way of implementing the BFGS method. Let $\mathcal{G}_{k}$ denote the subspace $\mathcal{G}_{k}=\operatorname{span}\left\{g_{0}, g_{1}, \ldots, g_{k}\right\}$, and let $\mathcal{G}_{k}^{\perp}$ denote the orthogonal complement of $\mathcal{G}_{k}$ in $\mathbb{R}^{n}$. Reduced-Hessian methods are based on the following result (see, e.g., Fletcher and Powell [11], Fenelon [8], and Siegel [33]).

Lemma 2.1. Consider the BFGS method applied to a general nonlinear function. If $H_{0}=\sigma I(\sigma>0)$ and $H_{k} p_{k}=-g_{k}$, then $p_{k} \in \mathcal{G}_{k}$ for all $k$. Moreover, if $z \in \mathcal{G}_{k}$ and $w \in \mathcal{G}_{k}^{\perp}$, then $H_{k} z \in \mathcal{G}_{k}$ and $H_{k} w=\sigma w$.

Let $r_{k}$ denote $\operatorname{dim}\left(\mathcal{G}_{k}\right)$, and let $B_{k}$ ( $B$ for "basis") denote an $n \times r_{k}$ matrix whose columns form a basis for $\mathcal{G}_{k}$. An orthonormal basis $Z_{k}$ can be defined from the QR decomposition $B_{k}=Z_{k} T_{k}$, where $T_{k}$ is a nonsingular upper-triangular matrix. Let the $n-r_{k}$ columns of $W_{k}$ define an orthonormal basis for $\mathcal{G}_{k}^{\perp}$. If $Q_{k}$ is the orthogonal matrix $Q_{k}=\left(\begin{array}{ll}Z_{k} & W_{k}\end{array}\right)$, then the transformation $x=Q_{k} x_{Q}$ defines a transformed approximate Hessian $Q_{k}^{T} H_{k} Q_{k}$ and a transformed gradient $Q_{k}^{T} g_{k}$. If $H_{0}=\sigma I(\sigma>0)$, it follows from (2.1) and Lemma 2.1 that the transformation induces a block-diagonal structure, with

$$
Q_{k}^{T} H_{k} Q_{k}=\left(\begin{array}{cc}
Z_{k}^{T} H_{k} Z_{k} & 0 \\
0 & \sigma I_{n-r_{k}}
\end{array}\right) \quad \text { and } \quad Q_{k}^{T} g_{k}=\left(\begin{array}{c}
Z_{k}^{T} g_{k} \\
0
\end{array}\right) .
$$


The positive-definite matrix $Z_{k}^{T} H_{k} Z_{k}$ is known as a reduced approximate Hessian (or just reduced Hessian). The vector $Z_{k}^{T} g_{k}$ is known as a reduced gradient.

If we write the equation for the search direction as $\left(Q_{k}^{T} H_{k} Q_{k}\right) Q_{k}^{T} p_{k}=-Q_{k}^{T} g_{k}$, it follows from (2.3) that

$$
p_{k}=Z_{k} q_{k}, \text { where } q_{k} \text { satisfies } Z_{k}^{T} H_{k} Z_{k} q_{k}=-Z_{k}^{T} g_{k} .
$$

If the Cholesky factorization $Z_{k}^{T} H_{k} Z_{k}=R_{k}^{T} R_{k}$ is known, $q_{k}$ can be computed from the forward substitution $R_{k}^{T} d_{k}=-Z_{k}^{T} g_{k}$ and back-substitution $R_{k} q_{k}=d_{k}$. The practical benefit of this approach is that, if $k \ll n$, the matrices $Z_{k}$ and $R_{k}$ require much less storage than $H_{k}$.

There are a number of alternative choices for $B_{k}$ (see Gill and Leonard [13, Theorem 2.3]). Both Fenelon and Siegel propose that $B_{k}$ be formed from a linearly independent subset of $\left\{g_{0}, g_{1}, \ldots, g_{k}\right\}$. With this choice, the orthonormal basis can be accumulated columnwise as the iterations proceed using Gram-Schmidt orthogonalization (see, e.g., Golub and Van Loan [18, pp. 218-220]). During iteration $k$, the number of columns of $Z_{k}$ either remains unchanged or increases by one, depending on the value of the scalar $\rho_{k+1}$ such that $\rho_{k+1}=\left\|\left(I-Z_{k} Z_{k}^{T}\right) g_{k+1}\right\|$. If $\rho_{k+1}=0$, the new gradient has no component outside range $\left(Z_{k}\right)$ and $g_{k+1}$ is said to be rejected. Thus, if $\rho_{k+1}=0, Z_{k}$ already provides a basis for $\mathcal{G}_{k+1}$ with $r_{k+1}=r_{k}$ and $Z_{k+1}=Z_{k}$. Otherwise, $r_{k+1}=r_{k}+1$ and the gradient $g_{k+1}$ is said to be accepted. In this case, $Z_{k}$ gains a new column $z_{k+1}$ defined by the identity $\rho_{k+1} z_{k+1}=\left(I-Z_{k} Z_{k}^{T}\right) g_{k+1}$. The calculation of $z_{k+1}$ also provides the $r_{k}$-vector $u_{k}=Z_{k}^{T} g_{k+1}$ and the scalar $z_{k+1}^{T} g_{k+1}$ $\left(=\rho_{k+1}\right)$, which are the components of the reduced gradient $Z_{k+1}^{T} g_{k+1}$ for the next iteration. For simplicity, we write $\left(Z_{k+1}, u_{k}, \rho_{k+1}, r_{k+1}\right)=\boldsymbol{o r t h o g}\left(Z_{k}, g_{k+1}, r_{k}\right)$ in the algorithms that follow. This orthogonalization procedure requires approximately $2 n r_{k}$ flops. Gram-Schmidt orthogonalization may be considered as an algorithm for computing the QR decomposition of $B_{k}$ without storing $T_{k}$. Suppose that at the start of iteration $k$ there exists a nonsingular $T_{k}$ with $B_{k}=Z_{k} T_{k}$. If $g_{k+1}$ is accepted, then

$$
B_{k+1}=\left(\begin{array}{ll}
B_{k} & g_{k+1}
\end{array}\right)=\left(\begin{array}{cc}
Z_{k} & z_{k+1}
\end{array}\right)\left(\begin{array}{cc}
T_{k} & Z_{k}^{T} g_{k+1} \\
0 & \rho_{k+1}
\end{array}\right)=Z_{k+1} T_{k+1}
$$

where the last equality defines $T_{k+1}$, which is nonsingular since $\rho_{k+1} \neq 0$. Otherwise, $T_{k+1}=T_{k}$.

Definition (2.4) of each search direction implies that $p_{j} \in \mathcal{G}_{k}$ for all $0 \leq j \leq k$. This leads naturally to another basis for $\mathcal{G}_{k}$ based on orthogonalizing the search directions $p_{0}, p_{1}, \ldots, p_{k}$. The next theorem implies that the columns of $Z_{k}$ constitute an orthonormal basis for $\mathcal{P}_{k}$, the span of all search directions $\left\{p_{0}, p_{1}, \ldots, p_{k}\right\}$ (for a proof, see Gill and Leonard [13]).

TheOREM 2.2. If $H_{0}=\sigma I(\sigma>0)$, then the subspaces $\mathcal{G}_{k}$ and $\mathcal{P}_{k}$ generated by the gradients and search directions of the conventional BFGS method are identical.

This result implies that $Z_{k}$ can be generated from either gradients or search directions, a point that will be used to advantage in section 3 .

Given $Z_{k+1}$ and $H_{k}$, the calculation of the search direction for the next iteration requires the Cholesky factor of $Z_{k+1}^{T} H_{k+1} Z_{k+1} \cdot{ }^{1}$ This factor can be obtained from $R_{k}$ in a two-step process without the need to know $H_{k}$. The first step, which is not

\footnotetext{
${ }^{1}$ As mentioned earlier, $H_{k+1}$ is usually $H_{k}^{\prime}$, which is defined by (2.1) However, we will implicitly alter $H_{k}^{\prime}$ further, as described in section 2.2 .
} 
needed if $g_{k+1}$ is rejected, is to compute the factor $R_{k}^{\prime}$ of $Z_{k+1}^{T} H_{k} Z_{k+1}$. This step involves adding a row and column to $R_{k}$ to account for the new last column of $Z_{k+1}$. It follows from Lemma 2.1 and (2.3) that

$$
Z_{k+1}^{T} H_{k} Z_{k+1}=\left(\begin{array}{cc}
Z_{k}^{T} H_{k} Z_{k} & Z_{k}^{T} H_{k} z_{k+1} \\
z_{k+1}^{T} H_{k} Z_{k} & z_{k+1}^{T} H_{k} z_{k+1}
\end{array}\right)=\left(\begin{array}{cc}
Z_{k}^{T} H_{k} Z_{k} & 0 \\
0 & \sigma
\end{array}\right)
$$

giving an expanded block-diagonal factor $R_{k}^{\prime}$ defined by

$$
R_{k}^{\prime}=\left\{\begin{array}{cl}
R_{k} & \text { if } r_{k+1}=r_{k} \\
\left(\begin{array}{cc}
R_{k} & 0 \\
0 & \sigma^{1 / 2}
\end{array}\right) & \text { if } r_{k+1}=r_{k}+1
\end{array}\right.
$$

The algorithm that defines $R_{k}^{\prime}$ from $R_{k}$ will be denoted by expand for obvious reasons. This expansion also involves vectors $v_{k}=Z_{k}^{T} g_{k}, u_{k}=Z_{k}^{T} g_{k+1}$, and $q_{k}=$ $Z_{k}^{T} p_{k}$, which are updated to give $v_{k}^{\prime}=Z_{k+1}^{T} g_{k}, u_{k}^{\prime}=Z_{k+1}^{T} g_{k+1}$, and $q_{k}^{\prime}=Z_{k+1}^{T} p_{k}$. As both $p_{k}$ and $g_{k}$ lie in range $\left(Z_{k}\right)$, if $g_{k+1}$ is accepted, the vectors $v_{k}^{\prime}$ and $q_{k}^{\prime}$ are trivially defined from $v_{k}$ and $q_{k}$ by appending a zero component (see (2.3)). Similarly, the vector $u_{k}^{\prime}$ is formed from $u_{k}$ and $\rho_{k+1}$. If $g_{k+1}$ is rejected, $v_{k}^{\prime}=v_{k}, u_{k}^{\prime}=u_{k}$, and $q_{k}^{\prime}=q_{k}$. In either case, $v_{k+1}$ is equal to $u_{k}^{\prime}$ and need not be calculated at the start of iteration $k+1$ (see Algorithm 2.1 below).

The second step of the modification alters $R_{k}^{\prime}$ to reflect the BFGS update to $H_{k}$. This update gives a modified factor $R_{k}^{\prime \prime}=\operatorname{update}\left(R_{k}^{\prime}, s_{k}, y_{k}\right)$, where $s_{k}=$ $Z_{k+1}^{T}\left(x_{k+1}-x_{k}\right)=\alpha_{k} q_{k}^{\prime}$, and $y_{k}=Z_{k+1}^{T}\left(g_{k+1}-g_{k}\right)=u_{k}^{\prime}-v_{k}^{\prime}$.

2.2. Reinitialization. The initial approximate Hessian can greatly influence the practical performance of quasi-Newton methods. The usual choice $H_{0}=\sigma I$ $(\sigma>0)$ can result in many iterations and function evaluations - especially if the iterates tend toward a minimizer at which the Hessian of $f$ is ill-conditioned (see, e.g., Powell [27] and Siegel [33]). This is sometimes associated with "stalling" of the iterates, a phenomenon that can greatly increase the overall cpu time for solution (or termination). The form of the transformed Hessian $Q_{k}^{T} H_{k} Q_{k}$ (see (2.3)) reveals the influence of $H_{0}$ on the approximate Hessian. In particular, the scale factor $\sigma$ represents the approximate curvature along all directions in $\mathcal{G}_{k}^{\perp}$. However, in the reduced-Hessian formulation, this initial approximate curvature is not installed until the end of iteration $k$, when it is used in the expand procedure according to (2.6). Our idea is to replace $\sigma$ whenever $g_{k+1}$ is accepted with a value more representative of the approximated curvature. This has the effect of reinitializing the approximate curvature along $z_{k+1}$ and is meant to alleviate inefficiencies resulting from poor choices of $H_{0}$. An estimate $\sigma_{k}$ of the approximate curvature is maintained and updated as new curvature information is obtained. Some popular choices for $\sigma_{k}$ are considered by Leonard [20, pp. 44-48]. In section 5 we discuss values that have been proposed for limited-memory methods.

The initial approximate curvature can be reinitialized by using some $\sigma_{k+1}$ in place of $\sigma_{k}$ in the expand procedure. Gill and Leonard [13] show that reinitialization can be done either before or after the expand, but they recommend the latter because it results in a simpler convergence result. Here, the reinitialization is performed after update to be consistent with that article. The procedure reinitialize involves simply changing the trailing diagonal element of $R_{k}^{\prime \prime}$ from $\sigma_{k}^{1 / 2}$ to $\sigma_{k+1}^{1 / 2}$ whenever $g_{k+1}$ is accepted. 
2.3. Summary. We conclude this section by defining a generic reduced-Hessian method that is the basis of the limited-memory method proposed in section 3. As described above, the reduced-Hessian method involves four main procedures: an orthogonalize, which determines $Z_{k+1}$ using the Gram-Schmidt QR process; an expand, which increases the order of the reduced Hessian by one; an update, which applies a BFGS update directly to the reduced Hessian; and a reinitialize, which reinitializes the last diagonal of the reduced Hessian factor.

Algorithm 2.1. (RHR) Reduced-Hessian method with Reinitialization.

Choose $x_{0}$ and $\sigma_{0}\left(\sigma_{0}>0\right)$;

$k=0 ; \quad r_{0}=1 ; \quad g_{0}=\nabla f\left(x_{0}\right)$;

$Z_{0}=\left(g_{0} /\left\|g_{0}\right\|\right) ; \quad R_{0}=\left(\sigma_{0}^{1 / 2}\right) ; \quad v_{0}=\left\|g_{0}\right\| ;$

while not converged do

Solve $R_{k}^{T} d_{k}=-v_{k} ; \quad R_{k} q_{k}=d_{k} ;$

$p_{k}=Z_{k} q_{k}$

Find $\alpha_{k}$ satisfying the Wolfe conditions (2.2);

$x_{k+1}=x_{k}+\alpha_{k} p_{k} ; \quad g_{k+1}=\nabla f\left(x_{k}+\alpha_{k} p_{k}\right) ;$

$\left(Z_{k+1}, u_{k}, \rho_{k+1}, r_{k+1}\right)=\operatorname{orthog}\left(Z_{k}, g_{k+1}, r_{k}\right)$;

$\left(R_{k}^{\prime}, u_{k}^{\prime}, v_{k}^{\prime}, q_{k}^{\prime}\right)=\operatorname{expand}\left(R_{k}, u_{k}, v_{k}, q_{k}, \rho_{k+1}, \sigma_{k}\right)$;

$s_{k}=\alpha_{k} q_{k}^{\prime} ; \quad y_{k}=u_{k}^{\prime}-v_{k}^{\prime} ; \quad R_{k}^{\prime \prime}=\operatorname{update}\left(R_{k}^{\prime}, s_{k}, y_{k}\right)$;

Compute $\sigma_{k+1} ; \quad R_{k+1}=$ reinitialize $\left(R_{k}^{\prime \prime}, \sigma_{k+1}\right)$;

$v_{k+1}=u_{k}^{\prime}$;

$k=k+1$

end do

When no reinitialization is done, this algorithm generates the same iterates as the conventional BFGS method with $H_{0}=\sigma_{0} I$. In exact arithmetic, the methods differ only in the storage needed and the number of operations per iteration. It can be shown that both with and without reinitialization, the algorithm retains two important properties of the BFGS method: it has quadratic termination, and it converges globally and Q-superlinearly on strongly convex functions (see Gill and Leonard [13]).

Algorithm RHR implicitly defines a full-sized BFGS approximate Hessian $H_{k}$. Let $Z_{k}$ and $R_{k}$ be defined at the start of the $k$ th iteration, and let $Q_{k}=\left(\begin{array}{ll}Z_{k} & W_{k}\end{array}\right)$ denote an orthogonal matrix whose first $r_{k}$ columns are the columns of $Z_{k}$. The full-sized approximate Hessian is given by

$$
H_{k}=Q_{k}\left(\begin{array}{cc}
R_{k}^{T} & 0 \\
0 & \sigma_{k}^{1 / 2} I_{n-r_{k}}
\end{array}\right)\left(\begin{array}{cc}
R_{k} & 0 \\
0 & \sigma_{k}^{1 / 2} I_{n-r_{k}}
\end{array}\right) Q_{k}^{T} .
$$

Given $R_{k}, Z_{k}$, and any $n$-vector $v$, the identity

$$
H_{k} v=Z_{k} R_{k}^{T} R_{k} Z_{k}^{T} v+\sigma_{k}\left(I-Z_{k} Z_{k}^{T}\right) v
$$

implies that products $H_{k} v$ can be calculated. This allows the reduced-Hessian approach to be used in constrained optimization algorithms that use $H_{k}$ as an operator via products of the form $H_{k} v$ (see, e.g., Gill, Murray, and Saunders [15]).

3. A limited-memory reduced-Hessian method. In this section we propose a limited-memory method that may be viewed as a reduced-Hessian method in which only the most recent curvature information is retained. As in Algorithm RHR, a 
triangular factor of the reduced Hessian is updated and reinitialized at each iterationthe crucial difference is that the number of basis vectors (and hence the dimension of the reduced Hessian) is limited by a preassigned value $m$. For problems with many variables, a choice of $m \ll n$ gives significant savings in storage compared to conventional quasi-Newton methods.

A simple limited-memory version of RHR can be defined by discarding the oldest gradient when the storage limit is reached. However, algorithms based on this idea have proved to be inefficient in practice. One explanation of this inefficiency is that discarding the oldest gradient invalidates RHR's property of finite termination on a quadratic function (see Theorem 3.1 and the concluding remarks of section 3.7). There is considerable numerical evidence that quadratic termination is beneficial when minimizing general functions; see, e.g., Siegel [31] and Leonard [20]. The limitedmemory method proposed here retains the property of finite termination by following Siegel's suggestion of using a basis of search directions rather than gradients. This strategy is sufficient to maintain quadratic termination when the oldest basis vector is discarded (see section 3.3).

An important feature of the method is that it is necessary to store and update the triangular factor $T_{k}$ associated with the orthogonal factorization $B_{k}=Z_{k} T_{k}$. In practice, we store $T_{k}$ and either $Z_{k}$ or $B_{k}$.

3.1. The search-direction basis and its factorization. We start by describing how the orthogonal factorization is maintained as directions are added to the basis. Initially, this procedure is described in the context of building an $m$-dimensional basis before a search direction is discarded, where $m$ is assumed to satisfy $m \geq 2$. The usual context is to add and remove a vector at every iteration. The procedure for removing a direction from the basis is described in section 3.2. To simplify the discussion, we assume that every gradient is accepted.

In order to allow for the fact that $Z_{k}$ is used in the equations that define $p_{k}$, the gradient $g_{k}$ is used as a temporary basis vector until it can be replaced by $p_{k}$. This implies that the $k$ th iteration involves three basis matrices: $B_{k}, B_{k}^{\prime}$, and $B_{k}^{\prime \prime}$. The starting basis is $B_{k}=\left(\begin{array}{llll}p_{0} & \cdots & p_{k-1} & g_{k}\end{array}\right)$. The matrix $B_{k}^{\prime}$ is obtained from $B_{k}$ by replacing $g_{k}$ by $p_{k}$ as soon as it is computed, and $B_{k}^{\prime \prime}$ is found by adding the accepted gradient to $B_{k}^{\prime}$. The matrices $B_{k}$ and $B_{k}^{\prime}$ differ by a single column, yet, by Theorem 2.2, their columns span the same subspace during the build process.

The procedure starts with $B_{0}=\left(g_{0}\right), T_{0}=\left(\left\|g_{0}\right\|\right)$, and $Z_{0}=\left(z_{0}\right)$, where $z_{0}=g_{0} /\left\|g_{0}\right\|$. Once $p_{0}$ is calculated, it is swapped into the basis to give $B_{0}^{\prime}=\left(p_{0}\right)$ and $T_{0}^{\prime}=\left(\left\|p_{0}\right\|\right)$. After the line search, $g_{1}$ is accepted (by assumption) and we define

$$
B_{0}^{\prime \prime}=\left(\begin{array}{ll}
p_{0} & g_{1}
\end{array}\right), \quad T_{0}^{\prime \prime}=\left(\begin{array}{cc}
\left\|p_{0}\right\| & u_{0} \\
0 & \rho_{1}
\end{array}\right), \quad \text { and } \quad Z_{0}^{\prime}=\left(\begin{array}{ll}
z_{0} & z_{1}
\end{array}\right)
$$

(see (2.5) and recall that $u_{k}=Z_{k}^{T} g_{k+1}$ ). With our assumption that $m \geq 2$, no vector need be discarded and these matrices define $B_{1}, T_{1}$, and $Z_{1}$. The $k$ th iteration $(1 \leq k \leq m-1)$ proceeds in a similar way, with

$$
B_{k}=\left(\begin{array}{llll}
p_{0} & \cdots & p_{k-1} & g_{k}
\end{array}\right), \quad T_{k}=\left(\begin{array}{ll}
\underline{T}_{k-1}^{\prime} & v_{k}
\end{array}\right), \quad \underline{T}_{k-1}^{\prime}=\left(\begin{array}{c}
T_{k-1}^{\prime} \\
0
\end{array}\right),
$$

and $Z_{k}=\left(\begin{array}{llll}z_{0} & \cdots & z_{k-1} & z_{k}\end{array}\right)$ (the form of the last column of $T_{k}$ follows from the definition of $v_{k}$ as $Z_{k}^{T} g_{k}$ ). Once $p_{k}$ is computed, it is swapped with $g_{k}$ in the basis 
with no computation required, yielding

$$
B_{k}^{\prime}=\left(\begin{array}{llll}
p_{0} & \cdots & p_{k-1} & p_{k}
\end{array}\right) \text { and } T_{k}^{\prime}=\left(\begin{array}{ll}
\underline{T}_{k-1}^{\prime} & q_{k}
\end{array}\right),
$$

where $q_{k}=Z_{k}^{T} p_{k}$. The matrix $Z_{k}$ is unchanged. While building the basis, the last component of $q_{k}$ is nonzero and the swap can always be done (see Leonard [20, pp. 9499]). After the line search, $g_{k+1}$ is accepted and the orthogonalization procedure yields

$$
B_{k}^{\prime \prime}=\left(\begin{array}{ll}
B_{k}^{\prime} & g_{k+1}
\end{array}\right)=\left(\begin{array}{lllll}
p_{0} & \cdots & p_{k-1} & p_{k} & g_{k+1}
\end{array}\right), \quad T_{k}^{\prime \prime}=\left(\begin{array}{cc}
T_{k}^{\prime} & u_{k} \\
0 & \rho_{k+1}
\end{array}\right),
$$

and $Z_{k}^{\prime}=\left(\begin{array}{lllll}z_{0} & \cdots & z_{k-1} & z_{k} & z_{k+1}\end{array}\right)$. These matrices are then passed to iteration $k+1$ as $B_{k+1}, T_{k+1}$, and $Z_{k+1}$, respectively.

3.2. Discarding the oldest basis vector. Now suppose that $k=m-1$. Given the assumption that every gradient is accepted, there are $m+1$ vectors in the basis at the end of this iteration. At this point, $p_{0}$, the oldest search direction, must be discarded before starting iteration $k+1$. This gives the new basis

$$
B_{k+1}=\left(\begin{array}{llll}
p_{1} & \cdots & p_{k} & g_{k+1}
\end{array}\right) .
$$

(On the other hand, if at least one gradient is rejected, then no vector is discarded at iteration $m$. In this case, $B_{k+1}$ contains $r_{k+1}\left(r_{k+1} \leq m\right)$ linearly independent vectors consisting of at most one gradient (the vector $g_{k+1}$ ) and a linearly independent set of search directions. If $g_{k+1}$ is rejected, $B_{k+1}$ will consist of $r_{k+1}$ linearly independent search directions.) Discarding a vector from the basis will decrease the rank by one. Hence, a symbol $r_{k}^{\prime}$ is needed for the intermediate rank determined by the orthogonalization procedure orthog. The final rank $r_{k+1}$ is then either $r_{k}^{\prime}-1$ or $r_{k}^{\prime}$ depending upon whether or not a basis vector is discarded.

When the oldest direction $p_{0}$ is discarded, the removal of its associated column from the basis must be reflected in all factorizations associated with $B_{k}^{\prime \prime}$. To simplify the description, the subscript $k$ is suppressed, and a bar is used to denote quantities with subscript $k+1$.

The relationship between the old and new bases $B^{\prime \prime}$ and $\bar{B}\left(=B_{k+1}\right)$ is given by $B^{\prime \prime}=\left(\begin{array}{ll}p_{0} & \bar{B}\end{array}\right)$, where $\bar{B}$ is $n \times m$. Associated with $\bar{B}$, we require $\bar{Z}$ and $\bar{T}$ such that $\bar{B}=\bar{Z} \bar{T}$. Moreover, the change from $Z^{\prime}$ to $\bar{Z}$ induces a corresponding change to the Cholesky factor. If $R^{\prime \prime \prime}$ denotes the factor defined by the reinitialize procedure, then we require the factor $\bar{R}$ such that $\bar{R}^{T} \bar{R}=\bar{Z}^{T} H^{\prime \prime} \bar{Z}$, where $H^{\prime \prime}$ is defined as in (2.7) but in terms of $\bar{\sigma}, Z^{\prime}$, and $R^{\prime \prime \prime}$. The matrix $H^{\prime \prime}$ is the $H_{k+1}$ defined in section 2.2 .

Daniel et al. [6] give the following method for updating $Z^{\prime}$ and $T^{\prime \prime}$. Given any orthogonal $S$, the orthogonal factorization of $B^{\prime \prime}$ may be written as $B^{\prime \prime}=Z^{\prime} T^{\prime \prime}=$ $Z^{\prime} S^{T} S T^{\prime \prime}=Z_{S} T_{S}$ (say). The matrix $S$ is constructed so that $T_{S}$ has the partitioned form

$$
T_{S}=\left(\begin{array}{cc}
t & \bar{T} \\
\tau & 0
\end{array}\right)
$$

where $\bar{T}$ is the desired $m \times m$ upper-triangular matrix, $t$ is an $m$-vector, and $\tau$ is a scalar. In particular, $S=P_{m, m+1} P_{m-1, m} \cdots P_{12}$, where $P_{i, i+1}$ is an $(m+1) \times(m+1)$ plane rotation in the $(i, i+1)$ plane that annihilates the $(i+1, i+1)$ element of $P_{i-1, i} \cdots P_{12} T^{\prime \prime}$. 
The matrix $\bar{Z}$ consists of the first $m$ columns of $Z_{S}$, i.e., $Z_{S}=\left(\begin{array}{ll}\bar{Z} & z\end{array}\right)$, where $z$ is an $n$-vector. From the definition of $B^{\prime \prime}$, we have

$$
B^{\prime \prime}=\left(\begin{array}{ll}
p_{0} & \bar{B}
\end{array}\right)=Z_{S} T_{S}=\left(\begin{array}{ll}
\bar{Z} t+\tau z & \bar{Z} \bar{T}
\end{array}\right),
$$

and it follows that $\bar{B}=\bar{Z} \bar{T}$ is the required orthogonal factorization.

Next, we propose how to update $R^{\prime \prime \prime}$ when the first column of $B^{\prime \prime}$ is discarded. The old and new orthogonal bases are related by the identity $\bar{Z}=Z_{s} E_{m}$, where $E_{m}$ comprises the first $m$ columns of the identity matrix of order $m+1$. From the definitions of $\bar{Z}$ and $H^{\prime \prime}$, the new reduced Hessian is given by

$$
\bar{Z}^{T} H^{\prime \prime} \bar{Z}=E_{m}^{T} Z_{S}^{T} H^{\prime \prime} Z_{S} E_{m}=E_{m}^{T} S Z^{\prime T} H^{\prime \prime} Z^{\prime} S^{T} E_{m}=E_{m}^{T} S R^{\prime \prime \prime T} R^{\prime \prime \prime} S^{T} E_{m}
$$

In general, $R^{\prime \prime \prime} S^{T}$ is not upper triangular, but it may be restored to upper-triangular form by a second sweep of plane rotations $\widetilde{S}$. The $(m+1) \times(m+1)$ matrix $\widetilde{S}$ is orthogonal and is chosen so that $\widetilde{S} R^{\prime \prime \prime} S^{T}$ is upper triangular. If $R_{S}=\widetilde{S} R^{\prime \prime \prime} S^{T}$ denotes the resulting product, then $\bar{Z}^{T} H^{\prime \prime} \bar{Z}=E_{m}^{T} R_{S}^{T} R_{S} E_{m}$, which implies that the leading $m \times m$ block of $R_{S}$ is the required factor $\bar{R}$. Note that at this point, we can define $\bar{H}\left(=H_{k+1}\right)$ in terms of $\bar{R}, \bar{Z}$, and $\bar{\sigma}$.

The matrix $\widetilde{S}$ is the product $\widetilde{P}_{m, m+1} \cdots \widetilde{P}_{23} \widetilde{P}_{12}$, where $\widetilde{P}_{i, i+1}$ is an $(m+1) \times$ $(m+1)$ plane rotation in the $(i, i+1)$ plane that annihilates the $(i, i+1)$ element of $\widetilde{P}_{i-1, i} \cdots \widetilde{P}_{12} R^{\prime \prime \prime} P_{12}^{T} \cdots P_{i, i+1}^{T}$. In practice, the two sweeps $\widetilde{S}$ and $S$ are interlaced so that only $\mathcal{O}\left(m^{2}\right)$ operations are required.

It remains to show how $u^{\prime}\left(=Z^{\prime T} \bar{g}\right)$ is updated, thereby avoiding the $m n$ operations necessary to compute the new reduced gradient $u^{\prime \prime}\left(=\bar{Z}^{T} \bar{g}\right)$ from scratch. The identity $u^{\prime \prime}=\bar{Z}^{T} \bar{g}=\left(Z_{S} E_{m}\right)^{T} \bar{g}=E_{m}^{T} S\left(Z^{\prime T} \bar{g}\right)=E_{m}^{T} S u^{\prime}$ implies that $u^{\prime \prime}$ comprises the first $m$ components of $S u^{\prime}$.

3.3. Comparison of the bases. We now revert to using subscripts to denote iteration indices. Under the assumption that every gradient is accepted, the gradient and search-direction bases at the start of iteration $m-1$ are given by $G_{m-1}=$ $\left(\begin{array}{llll}g_{0} & g_{1} & \cdots & g_{m-1}\end{array}\right)$ and $P_{m-1}=\left(\begin{array}{lllll}p_{0} & p_{1} & \cdots & p_{m-2} & g_{m-1}\end{array}\right)$. Theorem 2.2 implies that $\operatorname{range}\left(G_{m-1}\right)=\operatorname{range}\left(P_{m-1}\right)$, and we can expect that the value of $p_{m-1}$ is independent of the choice of basis. However, the following argument shows that this is not necessarily true for $p_{m}$, and hence the gradient and search-direction bases are not necessarily the same in the limited-memory context.

At the end of iteration $m-1$, both bases will have $m+1$ vectors. In the limited-memory context, the oldest basis vector must be discarded, giving bases $G_{m}=\left(\begin{array}{llll}g_{1} & g_{2} & \cdots & g_{m}\end{array}\right)$ and $P_{m}=\left(\begin{array}{lllll}p_{1} & p_{2} & \cdots & p_{m-1} & g_{m}\end{array}\right)$. These bases do not include $g_{0}$ and $p_{0}$ (which is parallel to $g_{0}$ ), respectively. Note that $p_{m-1}$ is not necessarily in range $\left(G_{m}\right)$ because $p_{m-1}$ may have a nonzero component of $g_{0}$, which has been discarded from the gradient basis. Since $p_{m-1} \in \operatorname{range}\left(P_{m}\right)$ by construction, it follows that range $\left(G_{m}\right) \neq \operatorname{range}\left(P_{m}\right)$. We will discuss a specific implication of this phenomenon in section 3.7.

3.4. An implicit representation of $Z$. When a basis vector is discarded, the application of the plane rotations to the right of $Z_{k}^{\prime}$ requires approximately $4 m n$ operations. Although it is possible to reduce this to $3 m n$ operations (see Daniel et al. [6]), the update to $Z_{k}^{\prime}$ dominates the time to perform an iteration and reduces the efficiency compared to other methods. For example, the total number of operations for an iteration of the limited-memory method of Nocedal [26] is approximately $4 m n$. 
Our limited-memory reduced-Hessian method is substantially faster if, as proposed by Siegel, the basis matrix $B_{k}$ is stored instead of $Z_{k}$. In this case, products involving $Z_{k}$ are computed as needed using $T_{k}$ and $B_{k}$, and the number of operations required to drop a column from the basis is reduced to $\mathcal{O}\left(r_{k}^{2}\right)$.

With an implicit definition of $Z_{k}$, the orthogonalization procedure becomes a method for updating the orthogonal factorization of $B_{k}$ without storing $Z_{k}$. Given $B_{k}$ and a new gradient $g_{k+1}$, the first step is to compute $u_{k}=Z_{k}^{T} g_{k+1}$ from the equations $T_{k}^{\prime T} u_{k}=B_{k}^{\prime T} g_{k+1}$. Once $u_{k}$ is known, $\rho_{k+1}$ can be computed from the identity $\rho_{k+1}^{2}=\left\|g_{k+1}\right\|^{2}-\left\|u_{k}\right\|^{2}$. The updated triangular factor $T_{k}^{\prime \prime}$ is defined by augmenting $T_{k}^{\prime}$ by a column formed from $u_{k}=Z_{k}^{T} g_{k+1}$ and $\rho_{k+1}$ (see (2.5)). Note that the column $z_{k+1}$ is not needed. The implicit form of $Z_{k}$ reduces the cost of the orthogonalization procedure by half to approximately $n r_{k}$ operations.

3.5. The limited-memory algorithm. We have described a reduced-Hessian limited-memory algorithm that needs three procedures in addition to those needed by Algorithm RHR: a swap, which replaces an accepted gradient $g_{k}$ with $p_{k}$ in the definition of $B_{k}$, giving a basis defined by $B_{k}^{\prime}$ and $T_{k}^{\prime}$; a new orthogonalize, which orthogonalizes $g_{k+1}$ with respect to $Z_{k}$, giving a new orthonormal basis defined by $B_{k}^{\prime \prime}$ and $T_{k}^{\prime \prime}$; and a discard, which drops the oldest search direction from the basis. As in Algorithm RHR, statements of the form $\left(B_{k}^{\prime}, T_{k}^{\prime}\right)=\boldsymbol{s w a p}\left(B_{k}, T_{k}\right)$ indicate computed quantities and their dependencies associated with a given procedure. Similarly, the results of the implicit orthogonalization and discard procedures are denoted by $\left(B_{k}^{\prime \prime}, T_{k}^{\prime \prime}, u_{k}, \rho_{k+1}, r_{k}^{\prime}\right)=\operatorname{iorthog}\left(B_{k}^{\prime}, T_{k}^{\prime}, g_{k+1}, r_{k}\right)$ and $\left(B_{k+1}, T_{k+1}, R_{k+1}, u_{k}^{\prime \prime}\right)=$ $\operatorname{drop}\left(B_{k}^{\prime \prime}, T_{k}^{\prime \prime}, R_{k}^{\prime \prime \prime}, u_{k}^{\prime}\right)$.

Algorithm 3.1. (L-RHR) Limited-memory version of Algorithm RHR.

Choose $x_{0}, \sigma_{0}\left(\sigma_{0}>0\right)$, and $m(m \geq 2)$;

$k=0 ; \quad r_{0}=1 ; g_{0}=\nabla f\left(x_{0}\right) ;$

$B_{0}=\left(g_{0}\right) ; \quad T_{0}=\left(\left\|g_{0}\right\|\right) ; \quad v_{0}=\left\|g_{0}\right\| ; \quad R_{0}=\left(\sigma_{0}^{1 / 2}\right) ;$

while not converged do

Solve $R_{k}^{T} d_{k}=-v_{k} ; \quad R_{k} q_{k}=d_{k} ;$

Solve $T_{k} w=q_{k} ; \quad p_{k}=B_{k} w ;$

if $g_{k}$ was accepted then $\left(B_{k}^{\prime}, T_{k}^{\prime}\right)=\boldsymbol{s w a p}\left(B_{k}, T_{k}\right)$;

Find $\alpha_{k}$ satisfying the Wolfe conditions (2.2);

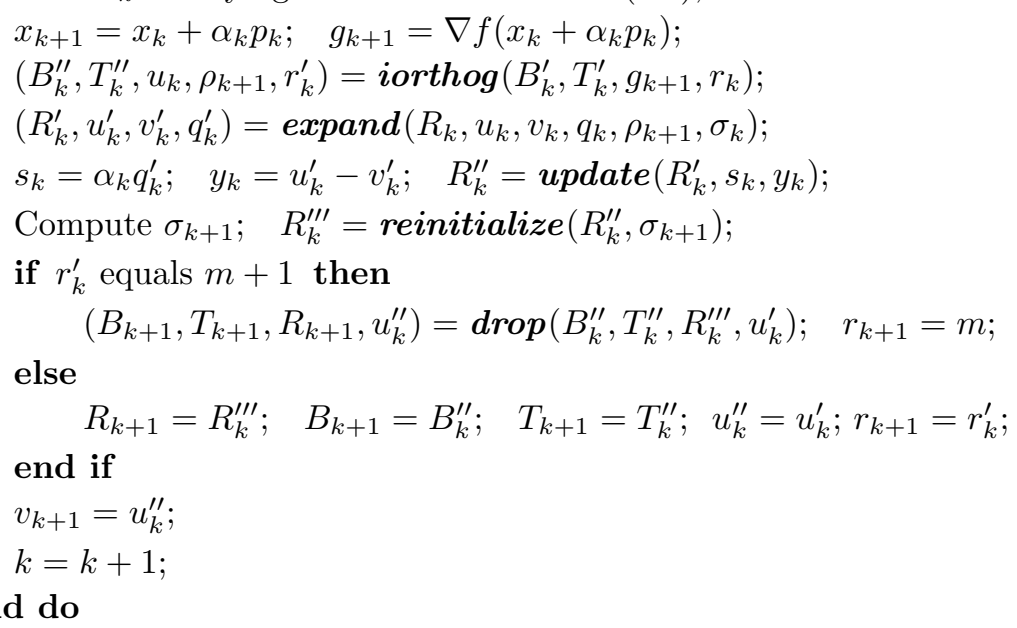


Iteration $k$ of Algorithm L-RHR requires $2 n r_{k}+2 n+\mathcal{O}\left(r_{k}^{2}\right)$ operations. (This total includes the work required for the swap, iorthog, update, and drop procedures but does not include any overhead incurred during the line search.)

3.6. Keeping the reduced Hessian vs. the reduced inverse Hessian. If Siegel's algorithm and an un-reinitialized version of L-RHR are applied with the same line search, then the same search directions and subspace bases are generated for the first $m$ iterations. During these iterations the full $n \times n$ Hessian of L-RHR (see (2.7)) is the inverse of the full inverse Hessian of Siegel's method. However, once a basis vector is discarded, the methods generate different search directions. In both algorithms, the updating procedures associated with a discard relegate curvature information associated with the oldest basis vector to the last row and column of their respective reduced matrices. The off-diagonal entries of this row and column are replaced by zero, and the diagonal element is set to either $\sigma$ or $1 / \sigma$ depending on the method. At this point the two basis matrices generate the same subspace, but because the leading $m \times m$ principal submatrices of a symmetric matrix and its inverse are not generally the inverse of each other, the full Hessian of L-RHR is no longer the inverse of the full inverse Hessian of Siegel's method. At the next iteration, the methods generate different search directions, and subsequent bases and reduced matrices are no longer related.

3.7. Finite termination on quadratics. Next we briefly discuss the properties of Algorithm L-RHR when it is applied with an exact line search to a strictly convex quadratic function.

THEOREM 3.1. Consider Algorithm L-RHR implemented with an exact line search and $\sigma_{0}=1$. If this algorithm is applied to a strictly convex quadratic function, then $R_{k}, B_{k}$, and $T_{k}(k \geq 1)$ satisfy

$$
R_{k}=\left(\begin{array}{ccccc}
a_{l} / h_{l} & b_{l} & 0 & \cdots & 0 \\
& a_{l+1} & b_{l+1} & \ddots & \vdots \\
& & \ddots & \ddots & 0 \\
& & & a_{k-1} & b_{k-1} \\
& & & & \sigma_{k}^{1 / 2}
\end{array}\right), \quad B_{k}=\left(\begin{array}{lllll}
p_{l} & p_{l+1} & \cdots & p_{k-1} & g_{k}
\end{array}\right)
$$

and

$$
T_{k}=\left(\begin{array}{cccccc}
h_{l} d_{l} & h_{l} t_{l, l+1} & h_{l} t_{l, l+2} & \cdots & h_{l} t_{l, k-1} & 0 \\
& d_{l+1} & t_{l+1, l+2} & \cdots & t_{l+1, k-1} & 0 \\
& & d_{l+2} & & \vdots & \vdots \\
& & & \ddots & t_{k-2, k-1} & 0 \\
& & & & d_{k-1} & 0 \\
& & & & & \left\|g_{k}\right\|
\end{array}\right),
$$

where $l=\max \{0, k-m+1\}$ and the scalars $a_{j}, b_{j}, t_{i j}, d_{j}$, and $h_{j}$ are given by

$$
a_{j}=\frac{\left\|g_{j}\right\|}{\left(y_{j}^{T} s_{j}\right)^{1 / 2}}, \quad b_{j}=-\frac{\left\|g_{j+1}\right\|}{\left(y_{j}^{T} s_{j}\right)^{1 / 2}}, \quad t_{i j}=-\frac{\left\|g_{j}\right\|^{2}}{\sigma_{j}\left\|g_{i}\right\|}, \quad d_{j}=-\frac{\left\|g_{j}\right\|}{\sigma_{j}}, \quad h_{j}=\delta_{j} \frac{\left\|p_{j}\right\| \sigma_{j}}{\left\|g_{j}\right\|}
$$


with $\delta_{j}=1$ if $j=0$, and $\delta_{j}=-1$ otherwise. Furthermore, the search directions are given by

$$
p_{0}=-g_{0} ; \quad p_{k}=-\frac{1}{\sigma_{k}} g_{k}+\beta_{k-1} p_{k-1}, \quad \beta_{k-1}=\frac{\sigma_{k-1}}{\sigma_{k}} \frac{\left\|g_{k}\right\|^{2}}{\left\|g_{k-1}\right\|^{2}}, \quad k \geq 1 .
$$

Proof. See Leonard [20].

COROLlary 3.2. If Algorithm L-RHR is used to minimize a strictly convex quadratic under the conditions of Theorem 3.1, then the method converges to the minimizer in at most $n$ iterations.

Proof. We show by induction that the search directions are parallel to the conjugate-gradient directions $\left\{d_{k}\right\}$. Specifically, $\sigma_{k} p_{k}=d_{k}$ for all $k$. This is true for $k=0$ since $1 \cdot p_{0}=-g_{0}=d_{0}$. Assume that $\sigma_{k-1} p_{k-1}=d_{k-1}$. Using Theorem 3.1 and the inductive hypothesis, we find

$$
\sigma_{k} p_{k}=-g_{k}+\sigma_{k-1} \frac{\left\|g_{k}\right\|^{2}}{\left\|g_{k-1}\right\|^{2}} p_{k-1}=-g_{k}+\frac{\left\|g_{k}\right\|^{2}}{\left\|g_{k-1}\right\|^{2}} d_{k-1}=d_{k},
$$

which completes the induction. The result now follows from the quadratic termination property of the conjugate-gradient method.

We remark that the specific form of L-RHR discussed in Theorem 3.1 defines a "rescaled" form of the classical Fletcher-Reeves conjugate-gradient method [12].

Let $p_{m}^{G}$ and $p_{m}^{P}$ denote the search directions defined during iteration $m$ of the gradient- and search-direction variants of the limited-memory algorithm. Observe that $p_{m-1}$ is parallel to $d_{m-1}$, regardless of which basis is used. However, since $g_{m} \in \operatorname{range}\left(G_{m}\right)$, but possibly $p_{m-1} \notin \operatorname{range}\left(G_{m}\right)$, the vector $p_{m}^{G}$ may not be parallel to $d_{m}$ and the gradient-basis variant does not have quadratic termination.

4. Implementation details. In this section, we describe some details associated with a particular implementation of Algorithm L-RHR. We outline a method for improving the orthonormal basis and discuss a practical criterion for accepting a gradient. We also provide information about the line search, the BFGS update, and restarts.

4.1. Reorthogonalization. For general applications the implicit QR version of $Z_{k}$ is recommended, with default memory size $m=5$. For larger values of $m$ (e.g., $m \geq 15$ ), it is often beneficial to use reorthogonalization in combination with the explicit QR. L-RHR employs the following reorthogonalization scheme proposed by Daniel et al. [6]. Let $u_{k}$ and $w_{k}$ denote the computed values of $Z_{k}^{T} g_{k+1}$ and $g_{k+1}-Z_{k} u_{k}$, respectively. The vectors $u_{k}$ and $w_{k}$ may be improved using one or more steps of the iterative refinement scheme:

$$
\Delta u_{k}=Z_{k}^{T} w_{k}, \quad u_{k} \leftarrow u_{k}+\Delta u_{k} ;
$$

and

$$
\Delta w_{k}=-Z_{k} \Delta u_{k}, \quad w_{k} \leftarrow w_{k}+\Delta w_{k} .
$$

L-RHR uses criteria suggested by Daniel et al. [6] for invoking and terminating the reorthogonalization. Each step of reorthogonalization adds approximately $2 n r_{k}$ operations to the cost of an iteration. Moreover, the use of an explicit $Z_{k}$ requires an additional $n r_{k}$ operations for the calculation of $z_{k+1}$ and an extra $3 n r_{k}$ operations when a basis vector is discarded (since the plane rotations associated with a discard must be applied to $Z_{k}$ as well as $T_{k}$ ). 
4.2. The criterion for gradient acceptance. In exact arithmetic, a gradient is accepted for the basis if $\rho_{k+1}>0$, where $\rho_{k+1}$ is the norm of $\left(I-Z_{k} Z_{k}^{T}\right) g_{k+1}$. This condition ensures that the basis vectors are linearly independent, and hence that $T_{k}^{\prime \prime}$ is nonsingular. When $\rho_{k+1}$ is computed in finite-precision, gradients with small (but nonzero) $\rho_{k+1}$ must be rejected to prevent $T_{k+1}$ and $B_{k+1}$ from being too illconditioned. In practice, an accepted gradient must satisfy $\rho_{k+1} \geq \epsilon\left\|g_{k+1}\right\|$, where $\epsilon$ is a preassigned positive constant. In the results of section $5, \epsilon$ was set to $10^{-4}$. Rounding error in the calculation of $\rho_{k+1}$ is exacerbated by the use of an implicit form for $Z_{k}$-for example, it is necessary to reject $g_{k+1}$ if the computed value of $\rho_{k+1}^{2}=\left\|g_{k+1}\right\|^{2}-\left\|u_{k}\right\|^{2}$ is negative. However, a negative computed value of $\rho_{k+1}^{2}$ rarely occurred in our experiments, and when it did, it did not prevent the method from terminating successfully (see section 5 for the criterion used). For example, a negative value was computed 268 times during the 69747 iterations in the runs of Table 5.6 below. Moreover, of the 10 problems in which a negative value occurred, all were solved successfully.

4.3. The line search, the BFGS update, and restarts. The line search is a slightly modified version of the one used in the package NPSOL [16]. It is designed to ensure that $\alpha_{k}$ satisfies the so-called strong Wolfe conditions,

$$
f\left(x_{k}+\alpha_{k} p_{k}\right) \leq f\left(x_{k}\right)+\mu \alpha_{k} g_{k}^{T} p_{k} \quad \text { and } \quad\left|g_{k+1}^{T} p_{k}\right| \leq \eta\left|g_{k}^{T} p_{k}\right|,
$$

where the constants $\mu$ and $\eta$ are chosen so that $0 \leq \mu<\eta<1$ and $\mu<\frac{1}{2}$ (see Gill et al. [16] or Fletcher [9, pp. 26-30]). The step-length parameters are $\mu=10^{-4}$ and $\eta=0.9$. The line search is based on using a safeguarded polynomial interpolation to find an approximate minimizer of the univariate function

$$
\phi_{k}(\alpha)=f\left(x_{k}+\alpha p_{k}\right)-f\left(x_{k}\right)-\mu \alpha g_{k}^{T} p_{k}
$$

(see Moré and Sorensen [23]). The step $\alpha_{k}$ is the first member of a minimizing sequence $\left\{\alpha_{k}^{i}\right\}$ that satisfies the Wolfe conditions. The sequence is usually started with $\alpha_{k}^{0}=1$ (see below).

If $\alpha_{k}$ satisfies the strong Wolfe conditions, it follows that $y_{k}^{T} s_{k} \geq-(1-\eta) g_{k}^{T} s_{k}>0$ and the BFGS update can be applied without difficulty. On very difficult problems, however, the combination of a poor search direction and rounding error in $f$ may prevent the line search from satisfying the line search conditions within 20 function evaluations. In this case, the search terminates with the step corresponding to the best value of $f$ found so far. If this $\alpha_{k}$ defines a strict decrease in $f$, the minimization continues. In this case, the BFGS update is skipped unless $y_{k}^{T} s_{k} \geq \epsilon_{M}\left|g_{k}^{T} s_{k}\right|$, where $\epsilon_{M}$ is the machine precision. If a strict decrease is not obtained after 20 function evaluations, the algorithm is restarted with $T_{k}=\left(\left\|g_{k}\right\|\right), v_{k}=\left\|g_{k}\right\|$, and $R_{k}=$ $\left(\sigma_{k}^{1 / 2}\right)$. To prevent the method from degenerating into steepest descent, no more restarts are allowed until the reduced Hessian has built up to its full size of $m$ rows and columns. In practice, a restart is rarely invoked. For example, in the experiments of Table 5.6, L-RHR used only one restart (on problem freuroth, which was not solved successfully). For comparison, L-BFGS-B used two restarts (on problem bdqrtic, again without success).

If $p_{k}$ is a poorly scaled version of the steepest-descent direction, the step to a minimizer of $\phi_{k}(\alpha)$ may be very small relative to one, and a large number of function evaluations may be needed to find an acceptable step length. To prevent this inefficiency, the initial step for the first line search and each line search immediately 
following a restart is limited so that $\alpha_{k}^{0} \leq \min \left\{\Delta /\left\|p_{k}\right\|, 1\right\}$, where $\Delta$ is a preassigned constant $(\Delta=2$ in the experiments described in the next section). This procedure ensures that the initial change in $x$ does not exceed $\Delta$.

5. Numerical results. In this section, we give numerical results for most of the large unconstrained problems in the $\mathrm{CUTE}^{2}$ collection (see Bongartz et al. [1]). After some discussion of the test problems, we compare L-RHR with and without reinitialization. Next, we illustrate the differences between L-RHR and Siegel's Algorithm 6 [33], which we refer to as ALG6. This is followed by results that compare L-RHR with L-BFGS and L-BFGS-B, which are two alternative implementations of the limitedmemory BFGS method. L-BFGS is based on an algorithm that maintains an implicit approximate inverse Hessian as a sequence of update pairs. L-BFGS-B employs an algorithm that updates an approximate Hessian in factored form $\theta I-W M W^{T}$, where $\theta$ is a scalar and $W M W^{T}$ is a matrix of low rank. L-BFGS-B is intended for problems with upper and lower bounds on the variables but is also recommended over L-BFGS-B for unconstrained problems (see Zhu et al. [34]).

Throughout, we use $m_{L B}$ to denote the number of update pairs to be kept in memory by L-BFGS and L-BFGS-B. This should not be confused with $m$, the number of vectors stored by L-RHR.

5.1. Test problem selection. The test set was constructed using the CUTE interactive select tool, which allows the identification of groups of problems with certain features. In our case, the select tool was first used to locate the twicecontinuously differentiable unconstrained problems for which the number of variables in the data file can be varied. Of these problems, the number of variables was set to a value in the range $100 \leq n \leq 1500$ according to criteria that we discuss below. The input for the select tool was as follows:

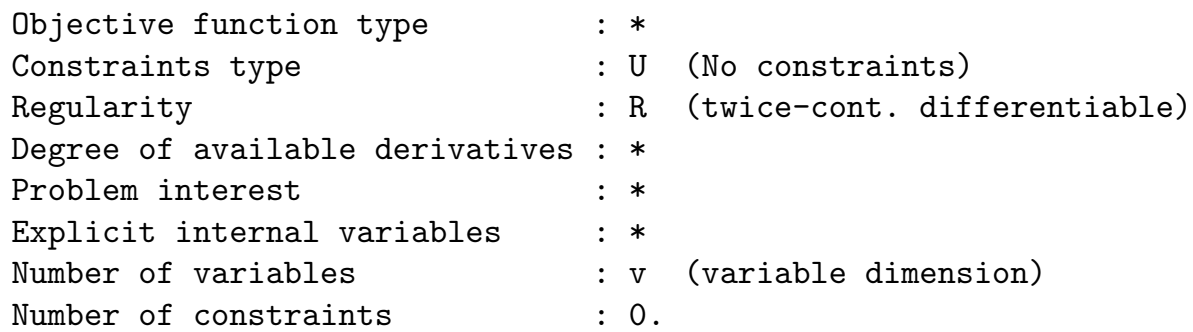

A total of 87 problems was obtained from this selection. Six fixed-dimension problems were obtained by using the select tool with the number of variables set as follows:
Number of variables
: in $[50,1000]$.

Additional criteria were used to determine the suitability of these 93 problems, as we now explain.

After using the select tool, it remained to determine a suitable value of $n$ for the problems with variable dimension. The value $n=1500$ was used for the twelve problems dixmaana-dixmaanl, as suggested by Zhu et al. [34]. Values $n \approx 1000$ were used for most of the remaining problems, but it was necessary to choose significantly smaller values of $n$ in some cases. The problems chnrosnb, errinros, and watson have limits on the size of $n$, and the mandated maximum values of 50,50, and 31, respectively, were used in these cases. It was also necessary to limit $n$ to be less than

\footnotetext{
${ }^{2}$ The version of CUTE used was obtained September 7, 2001.
} 
1000 if a problem could not be decoded using the CUTE decoder sifdec (compiled with the option tobig). For any such problem, the values $n=300$ and $n=100$ were tried successively until the decoding succeeded. Problems in this category were arglina-arglinc, brownal, hilberta, hilbertb, mancino, penalty3, and sensors. The value $n=300$ was used for arglina, brownal, hilberta, and hilbertb. The value $n=100$ was used for mancino, penalty3, and sensors. The problems arglinb $(n=300)$ and arglinc $(n=100)$ and penalty3 $(n=100)$ were successfully decoded but were removed from the set for reasons described below.

A value of $n$ such that $n<1000$ was also used if both L-BFGS-B and L-RHR failed to meet the termination criterion with $m, m_{L B}=5,15,30$, and 45 . (The termination criterion will not be satisfied if there is a failure in the line search or 40, 000 iterations are completed.) In this case, 300 and 100 were tried successively to determine an acceptable value for $n$. The problems arglinb, arglinc, curly10, curly20, curly30, fletchbv, hydc20ls (fixed $n=99$ ), indef, nonmsqrt, penalty3, sbrybnd, scosine, scurly10, scurly20, and scurly30 were removed from the test set since, even with $n=100$, neither method could meet the termination criterion. The value $n=100$ was used for penalty2 since neither L-RHR nor L-BFGS-B could achieve the termination criterion with $n=1000$ or $n=300$.

These selection criteria had the effect of removing 15 problems from the list generated by the select tool. This left 78 problems suitable for testing. For completeness, we list the problems not already mentioned, with their associated values of $n$. There were 44 variable-dimension problems with $n=1000$ : arwhead, bdqrtic, broydn7d, brybnd, chainwoo, cosine, cragglvy, dixon3dq, dqdrtic, dqrtic, edensch, engval1, extrosnb, fletcbv2, fletcbv3, fletchcr, freuroth, genhumps, genrose, liarwhd, morebv, ncb20, ncb20b, noncvxu2, noncvxun, nondia, nondquar, penalty1, powellsg, power, quartc, schmvett, sinquad, sparsine, sparsqur, spmsrtls, srosenbr, testquad, tointgss, tquartic, tridia, vardim, vareigvl, and woods. There were four problems with $n=1024$ : fminsrf2, fminsurf, msqrtals, and msqrtbls. The remaining three variabledimension problems were eigenals $(n=1056)$, eigenbls $(n=1056)$, and eigencls $(n=1122)$. Finally, the names and numbers of variables of the five fixed-dimension problems included in the test set were deconvu $(n=61)$, eg2 $(n=1000)$, tointgor $(n=50)$, tointpsp $(n=50)$, and tointqor $(n=50)$.

All runs were made on a Sun UltraSPARC-IIi (single cpu at 333MHz) with $256 \mathrm{MB}$ of RAM. The algorithms L-RHR, L-BFGS-B, and L-BFGS are coded in Fortran and were compiled using g77. ALG6 is coded in $\mathrm{C}$ and was compiled using gcc. Full compiler optimization was used in all cases. The caption of each table specifies the amount of limited memory used and indicates whether or not reinitialization and/or reorthogonalization was used. All methods were terminated when $\left\|g_{k}\right\|_{\infty}<10^{-5}$, as proposed by Zhu et al. [34].

5.2. L-RHR with and without reinitialization. Table 5.1 gives the results of running L-RHR both with and without reinitialization. Without reinitialization, the parameter $\sigma$ was fixed at $y_{0}^{T} s_{0} /\left\|s_{0}\right\|^{2}$ (see (2.6)). ${ }^{3}$ This is the scheme proposed by Siegel [33]. With reinitialization, $\sigma_{0}=1$ and $\sigma_{k}=y_{k}^{T} y_{k} / y_{k}^{T} s_{k}(k \geq 1)$, which are the reciprocals of the parameters used by Liu and Nocedal [21]. Of the 78 problems attempted, L-RHR with reinitialization solved 74 problems satisfactorily and reduced the gradient to within two orders of magnitude of the $10^{-5}$ target value on three

\footnotetext{
${ }^{3}$ The steepest-descent direction is used for the first iteration. After the first step, $\sigma$ is set to $y_{0}^{T} s_{0} /\left\|s_{0}\right\|^{2}$ and $R$ is defined accordingly.
} 
others (bdqrtic, freuroth, and noncvxun). The algorithm was unable to reduce $\left\|g_{k}\right\|_{\infty}$ below $1.5 \times 10^{-2}$ for fletcbv3. Without reinitialization, L-RHR was able to solve only 70 problems and required considerably more function and gradient evaluations on almost every problem attempted. (The additional four unsolved problems were chainwoo, cragglvy, edensch, and penalty2.) Table 5.1 gives the total number of iterations, function evaluations and cpu seconds for L-RHR with and without reinitialization on the 70 problems that could be solved by both versions. These results indicate that reinitialization provides substantial practical benefits and indicates an advantage of L-RHR compared to Siegel's method, which does not include reinitialization. A direct comparison between L-RHR and Siegel's method is given in the next section.

TABLE 5.1

$\mathrm{L}-\mathrm{RHR}^{\mathrm{a}}$ with and without reinitialization on 70 CUTE problems.

\begin{tabular}{|l|c|r|c|c|}
\hline \multicolumn{1}{|c|}{ L-RHR } & Itns & Fncs & Cpu & Fail \\
\hline with reinitialization & 65115 & 66914 & 1567 & 4 \\
without reinitialization & 83611 & 107253 & 1884 & 8 \\
\hline
\end{tabular}

${ }^{\text {a }} m=5$; without reorthogonalization.

5.3. L-RHR compared with ALG6. Next we compare L-RHR with Siegel's ALG6. In the first set of runs, ALG6 uses the recommended value of $\epsilon=10^{-3}$ for the gradient acceptance parameter (see [32, p. 8]). The line search in ALG6 is a slightly modified version of the one in Powell's Fortran package TOLMIN [28]. It attempts to satisfy the Wolfe conditions (see (2.2)) with $\mu=10^{-2}$ and $\eta=0.9$ but allows $f\left(x_{k+1}\right) \geq f\left(x_{k}\right)$ to within a small tolerance. ALG6 is not optimized for cpu time, and it may be possible to improve the performance by making appropriate changes to the code. However, the relative differences in cpu times are unlikely to be altered by recoding because many of the run times are dominated by the cumulative cost of the function evaluations (see section 5.5).

Table 5.2 gives the results of comparing ALG6 with a version of L-RHR implemented without reinitialization. Algorithm L-RHR succeeded on 70 of the 78 test problems and reduced the gradient norm to at most $10^{-3}$ on seven others: bdqrtic, chainwoo, cragglvy, edensch, freuroth, noncvxun, and penalty2. On the other unsuccessful case, fletcbv3, the final gradient norm was $1.1 \times 10^{-1}$. ALG6 succeeded on 74 out of the 78 problems. On arwhead, bdqrtic, and noncvxun, ALG6 was able to reduce the gradient norm to at most $10^{-3}$. On fletcbv3, ALG6 reduced the gradient norm to $4.7 \times 10^{-2}$. Table 5.2 summarizes the results for the 69 problems that both methods were able to solve successfully. If the L-RHR line search is made to conform to ALG6 by allowing $f\left(x_{k+1}\right) \geq f\left(x_{k}\right)$ to within a prescribed tolerance, then L-RHR is able to solve three more problems, chainwoo, edensch, and penalty2.

TABLE 5.2

$\mathrm{L}-\mathrm{RHR}^{\mathrm{a}}$ compared with $\mathrm{ALG}^{\mathrm{b}}$ on 69 CUTE problems.

\begin{tabular}{|l|r|c|c|c|}
\hline Method & \multicolumn{1}{|c|}{ Itns } & Fncs & Cpu & Fail \\
\hline L-RHR & 83601 & 107237 & 1884 & 8 \\
ALG6 & 101959 & 194091 & 5744 & 4 \\
\hline
\end{tabular}

${ }^{\text {a }} m=5$; with no reinitialization and no reorthogonalization; $\epsilon=10^{-4}$.

${ }^{\mathrm{b}} m=5$; with Siegel's version of the TOLMIN line search; $\epsilon=10^{-3}$. 
Since the L-RHR and ALG6 directions have similar definitions when L-RHR does not use reinitialization, it might seem surprising that L-RHR requires significantly fewer function evaluations than ALG6. This phenomenon can be partly explained by differences in the line search and the different choice of $\epsilon$. To illustrate these effects, Table 5.3 gives a comparison between L-RHR and ALG6 when both algorithms are implemented with the NPSOL line search and $\epsilon=10^{-4}$. Note that the number of function evaluations for ALG6 decreases dramatically, though the stricter requirement that $f\left(x_{k+1}\right)<f\left(x_{k}\right)$ results in a few more failures.

TABLE 5.3

$\mathrm{L}-\mathrm{RHR}^{\mathrm{a}}$ compared with ALG6 ${ }^{\mathrm{b}}$ on 69 CUTE problems.

\begin{tabular}{|l|c|c|c|c|}
\hline Method & Itns & Fncs & Cpu & Fail \\
\hline L-RHR & 82363 & 105997 & 1884 & 8 \\
ALG6 & 86970 & 138512 & 3620 & 8 \\
\hline
\end{tabular}

${ }^{\text {a }} m=5$; with no reinitialization and no reorthogonalization; $\epsilon=10^{-4}$.

${ }^{\mathrm{b}} m=5$; with the line search from NPSOL; $\epsilon=10^{-4}$.

The results are closer, but Table 5.3 illustrates that the methods are still generating different iterates. This is because, in the limited-memory context, an algorithm based on updating a reduced Hessian is fundamentally different from an algorithm based on updating a reduced inverse Hessian. The directions generated by ALG6 and an un-reinitialized version of L-RHR are only the same until a basis vector is discarded. From this point, the Hessian of L-RHR is no longer related to the inverse Hessian of ALG6 (see section 3.6). For example, Table 5.4 illustrates that if L-RHR and ALG6 are applied to problem msqrtals with $m=30$, the function values and gradient norms are in close agreement at iteration 30. At the next iteration the first discard is made and most of the agreement is lost. By iteration 50, only 1 significant digit of agreement remains. The total numbers of iterations required are 2556 and 3133 for L-RHR and ALG6, respectively. It follows that L-RHR's significant advantage in the "Fncs" column of Table 5.3 results from the use of a reduced Hessian instead of a reduced inverse Hessian.

TABLE 5.4

${\mathrm{L}-\mathrm{RHR}^{\mathrm{a}}}^{\mathrm{a}}$ and $\mathrm{ALG6}^{\mathrm{b}}$ applied to msqrtals with $m=30$.

\begin{tabular}{|c|c|c|c|c|}
\hline \multirow{2}{*}{$k$} & $f_{k}$ & \multicolumn{2}{c|}{ ALG6 } \\
\cline { 2 - 5 } & & $\left\|g_{k}\right\|_{\infty}$ & $f_{k}$ & $\left\|g_{k}\right\|_{\infty}$ \\
\hline 30 & 0.387222166177969 & 0.583990052575414 & 0.387222166177971 & 0.583990052575427 \\
31 & 0.341636067001799 & 0.569093782828364 & 0.341694835004789 & 0.569019238515868 \\
50 & 0.096802831009406 & 0.126889757489794 & 0.097640019163964 & 0.140754040816826 \\
\hline
\end{tabular}

${ }^{\text {a }} m=5$; with no reinitialization and no reorthogonalization; $\epsilon=10^{-4}$.

${ }^{\mathrm{b}} m=5$; with the line search from NPSOL; $\epsilon=10^{-4}$.

We provide one final comparison in which L-RHR uses reinitialization. In this case, both L-RHR and ALG6 succeed on 74 problems, and there are 73 problems that are solved by both methods. Table 5.5 shows the overall results for these 73 problems. Note that, overall, the use of reinitialization by L-RHR results in significantly fewer function evaluations compared to ALG6. 
TABle 5.5

$\mathrm{L}-\mathrm{RHR}^{\mathrm{a}}$ compared with ALG6 ${ }^{\mathrm{b}}$ on 73 CUTE problems.

\begin{tabular}{|l|c|r|c|c|}
\hline Method & \multicolumn{1}{|c|}{ Itns } & \multicolumn{1}{c|}{ Fncs } & Cpu & Fail \\
\hline L-RHR & 69737 & 71782 & 1592 & 4 \\
ALG6 & 103217 & 195405 & 5752 & 4 \\
\hline
\end{tabular}

${ }^{\text {a }} m=5$; with reinitialization; without reorthogonalization.

b $m=5$; with Siegel's implementation of the TOLMIN line search.

5.4. L-RHR compared with L-BFGS-B. L-RHR and Seigel's method are related to the limited-memory BFGS method of Byrd et al. [4] because all three methods consolidate the quasi-Newton updates into dense matrices. By contrast, L-BFGS keeps an implicit inverse Hessian by storing a fixed number of vector pairs $\left(\gamma_{k}, \delta_{k}\right)$ (see (2.1) for the definitions of $\gamma_{k}$ and $\delta_{k}$ ). Products of the inverse Hessian with a vector are then formed without the need to keep an explicit $H_{k}$ (see Nocedal [26]). Consolidation of the updates is crucial for efficiency if a limited-memory method is to be extended to handle upper and lower bounds on the variables. In this section we compare L-RHR with Version 2.1 of the code L-BFGS-B (see Zhu et al. [34]), which is an implementation of the method of Byrd, Lu, Nocedal and Zhu. L-BFGS-B applies the strong Wolfe conditions (4.1) using the line search of Moré and Thuente [24] with line search parameters $\mu=10^{-4}$ and $\eta=0.9$. The memory for L-BFGS-B was limited to $m_{L B}=5$ pairs of vectors, which is twice the storage used by L-RHR.

Table 5.6 summarizes the performance of L-RHR and L-BFGS-B on the 74 CUTE problems on which both methods succeed. On these 74 problems, L-RHR requires fewer function evaluations than L-BFGS-B on 27 problems and more function evaluations on 43 problems. L-RHR requires less cpu time than L-BFGS-B on 55 problems and more cpu time than L-BFGS-B on 16 problems. L-BFGS-B was able to solve one more problem than L-RHR, namely, fletcbv3 (L-RHR reduced the gradient norm to $1.5 \times 10^{-2}$ in this case). Neither method was able to satisfy the termination criterion on bdqrtic, freuroth, and noncvxun. In these cases, the final gradient norms for L-RHR (L-BFGS-B) were $2.90 \times 10^{-4}\left(6.08 \times 10^{-4}\right), 3.40 \times 10^{-4}\left(1.60 \times 10^{-5}\right)$, and $1.00 \times 10^{-3}$ $\left(1.66 \times 10^{-3}\right)$, respectively. Overall, L-RHR requires a comparable number of function evaluations and has a significant advantage in terms of cpu time.

TABLE 5.6

$\mathrm{L}-\mathrm{RHR}^{\mathrm{a}}$ and $\mathrm{L}-\mathrm{BFGS}-\mathrm{B}^{\mathrm{b}}$ on 74 CUTE problems.

\begin{tabular}{|l|c|c|c|c|}
\hline \multicolumn{1}{|c|}{ Method } & Itns & Fcns & Cpu & Fail \\
\hline L-RHR & 69747 & 71798 & 1592 & 4 \\
L-BFGS-B & 66717 & 72264 & 1916 & 3 \\
\hline
\end{tabular}

${ }^{\text {a }} m=5 n$-vectors; with reinitialization; without reorthogonalization.

${ }^{\mathrm{b}} m_{L B}=5$ pairs of $n$-vectors.

Although the values $m=5$ and $m_{L B}=5$ are recommended for L-RHR and L-BFGS-B, it is of interest to investigate the relative performance of the algorithms as the memory size is increased. For example, the overhead required to solve an unsymmetric $2 m_{L B} \times 2 m_{L B}$ system every iteration of L-BFGS-B might suggest that L-RHR would have a greater cpu time advantage as $m$ and $m_{L B}$ are increased. Table 5.7 gives the performance of L-RHR and L-BFGS-B with increasing memory-size parameters $m$ and $m_{L B}$. When $m \geq 15$ it is recommended that L-RHR uses reorthogonalization to maintain a good basis and improve robustness (see section 4.1). However, to illustrate 
the difference when L-RHR with $m=5$ does and does not use reorthogonalization, the results of Table 5.7 use reorthogonalization for all memory sizes. For $m=5,15$, 30 , and 45 , the numbers of failures for L-RHR without reorthogonalization are 4, 5, 7 , and 11 , respectively. With reorthogonalization, these numbers drop to $4,4,3$, and 3 , respectively. Table 5.7 provides the total number of reorthogonalizations required for each value of $m$. Although the amount of work per iteration is more than doubled with reorthogonalization, the cpu seconds required for $m=5$ decreases from 1590 to 1580. In this case, the reductions in iterations and function evaluations compensates for the increased cost of computing the search direction.

All 78 problems were attempted, but the totals in each row include only the statistics for problems that could be solved by both methods. The cpu time advantage for L-RHR increases from $82 \%$ of the time required by L-BFGS-B to $59 \%$ as $m$ and $m_{L B}$ are increased from 5 to 45 . However, L-BFGS-B gains an advantage in terms of the number of function evaluations. The interpretation of these results is complicated by the sharp increase in function evaluations when $m$ is increased from 15 to 30 . This increase occurs because both methods are able to solve problem noncvxun when $m=30$. If noncvxun is removed from the problem set, a total of $69,522(72,264)$, $67,293(62,470), 64,808(57,114)$, and $58,075(54,391)$ evaluations are required by L-RHR (L-BFGS-B) for $m\left(m_{L B}\right)$ with the values $5,15,30$, and 45 , respectively. These results indicate that for both methods, the number of function evaluations generally decreases as the limited-memory size is increased.

TABLE 5.7

$\mathrm{L}-\mathrm{RHR}^{\mathrm{a}}$ and L-BFGS-B with various memory sizes for 78 CUTE problems.

\begin{tabular}{|c|crccc|ccccc|}
\hline \multirow{2}{*}{ Mem } & \multicolumn{5}{|c|}{ L-RHR } & \multicolumn{4}{c|}{ L-BFGS-B } \\
\cline { 2 - 10 } & Itns & Fcns & Reors & Cpu & Fail & Itns & Fcns & Cpu & Fail \\
\hline 5 & 67573 & 69522 & 42018 & 1580 & 4 & 66717 & 72264 & 1916 & 3 \\
15 & 65050 & 67293 & 57662 & 1951 & 4 & 57302 & 62470 & 2155 & 5 \\
30 & 99122 & 101432 & 95445 & 2866 & 3 & 80669 & 86304 & 3744 & 2 \\
45 & 78344 & 80550 & 76173 & 2782 & 3 & 65859 & 70962 & 4686 & 3 \\
\hline
\end{tabular}

${ }^{a}$ With reinitialization and reorthogonalization.

5.5. L-RHR compared with L-BFGS. We conclude this section by providing a comparison of L-RHR with L-BFGS, which is a limited-memory BFGS method that maintains an implicit inverse approximate Hessian as a sequence of $m_{L B}$ update pairs (see Nocedal [26] and Liu and Nocedal [21]). The recommended memory size is $m_{L B}=5$. The search direction requires approximately $4 n m_{L B}$ operations, which is roughly twice the work required by L-RHR. L-BFGS uses the Moré and Thuente line search with the same parameter settings as L-BFGS-B.

Table 5.8 summarizes the performance of L-RHR and L-BFGS on the 74 problems that both methods solved successfully. Of these 74 problems, L-RHR required fewer function evaluations on 27 problems and L-BFGS required fewer evaluations on 43 problems. L-RHR required less cpu time on 30 problems and more time on 41 problems. Overall, L-BFGS and L-RHR required comparable numbers of function evaluations and iterations. However, even though L-RHR requires roughly half as much work to compute the search direction, the overall cpu time was very close to that of L-BFGS. Further investigation using a performance profiler indicated that the cost of evaluating the objective for seven of the problems (eigenals, eigenbls, eigencls, msqrtals, msqrtbls, ncb20, and ncb20b) dominated the overall computation time. The 
cpu time required by L-RHR to compute the search direction was less than $60 \%$ of that needed by L-BFGS. However, on these seven critical problems, the calculation of the search direction constitutes less than $10 \%$ of the solve time.

TABLE 5.8

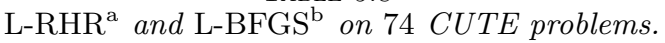

\begin{tabular}{|l|c|c|c|c|}
\hline Method & Itns & Fcns & Cpu & Fail \\
\hline L-RHR & 69747 & 71798 & 1592 & 4 \\
L-BFGS & 66445 & 71978 & 1670 & 4 \\
\hline
\end{tabular}

${ }^{a} m=5 n$-vectors; with reinitialization; without reorthogonalization.

${ }^{\text {b }} m_{L B}=5$ pairs of $n$-vectors.

In order to compare L-RHR with L-BFGS as $m$ and $m_{L B}$ are increased, L-RHR uses reorthogonalization for improved robustness. As $m$ and $m_{L B}$ take on the values $5,15,30$, and 45 , the total function evaluations on the problems solved by both methods L-RHR (L-BFGS) are 69,522 (71,978), 67,293 (63,466), 101,432 (80,832), and $80,550(88,717)$. As in the comparison with L-BFGS-B, L-RHR is competitive in terms of function evaluations, but in this case L-RHR requires more cpu time when $m \geq 15$. Although some of the functions dominate the overall cpu time when $m=5$, their evaluation carries less weight with increasing $m$. This effect, when combined with the cost of reorthogonalization, is why L-RHR is slower than L-BFGS when $m=45$, even though L-RHR requires significantly fewer function evaluations. We reemphasize that L-RHR requires approximately half the storage of L-BFGS.

6. Summary and conclusions. We have presented theoretical and practical details of a limited-memory reduced-Hessian method for large-scale smooth unconstrained optimization problems for which first derivatives are available. The method maintains the Cholesky factor of a reduced Hessian and requires roughly half the storage of conventional limited-memory methods.

The numerical results of Section 5 confirm that L-RHR is efficient and reliable on a set of large test problems from the CUTE collection. Moreover, it is shown that Hessian reinitialization and selective reorthogonalization are vital components of an efficient and robust reduced-Hessian method.

When compared to the state-of-the-art code L-BFGS-B on our test set, L-RHR converges in less cpu time, while requiring comparable numbers of function evaluations. Compared to the code L-BFGS, L-RHR required comparable numbers of function evaluations and iterations when both algorithms were applied with their default memory sizes.

Acknowledgments. We thank Dirk Siegel for graciously providing a copy of his limited-memory code. We also appreciate many suggestions from the referees and Associate Editor Jorge Nocedal.

\section{REFERENCES}

[1] I. Bongartz, A. R. Conn, N. I. M. Gould, And P. L. Toint, CUTE: Constrained and unconstrained testing environment, ACM Trans. Math. Software, 21 (1995), pp. 123-160.

[2] A. G. Buckley, A combined conjugate-gradient quasi-Newton minimization algorithm, Math. Program., 15 (1978), pp. 200-210.

[3] - Extending the relationship between the conjugate-gradient and BFGS algorithms, Math. Program., 15 (1978), pp. 343-348.

[4] R. H. Byrd, P. Lu, J. Nocedal, AND C. Zhu, A limited memory algorithm for bound constrained optimization, SIAM J. Sci. Comput., 16 (1995), pp. 1190-1208. 
[5] R. H. Byrd, J. Nocedal, And R. B. Schnabel, Representations of quasi-Newton matrices and their use in limited-memory methods, Math. Program., 63 (1994), pp. 129-156.

[6] J. W. Daniel, W. B. Gragg, L. Kaufman, and G. W. Stewart, Reorthogonalization and stable algorithms for updating the Gram-Schmidt QR factorization, Math. Comput., 30 (1976), pp. 772-795.

[7] J. E. Dennis, JR. And R. B. Schnabel, A new derivation of symmetric positive definite secant updates, in Nonlinear programming, 4 (Proc. Sympos., Special Interest Group on Math. Programming, Univ. Wisconsin, Madison, Wis., 1980), Academic Press, New York, 1981, pp. $167-199$.

[8] M. C. Fenelon, Preconditioned Conjugate-Gradient-Type Methods for Large-Scale Unconstrained Optimization, PhD thesis, Department of Operations Research, Stanford University, Stanford, CA, 1981.

[9] R. Fletcher, Practical Methods of Optimization, John Wiley and Sons, Chichester and New York, second ed., 1987.

[10] — An optimal positive definite update for sparse Hessian matrices, SIAM J. Optim., 5 (1995), pp. 192-218.

[11] R. Fletcher and M. J. D. Powell, A rapidly convergent descent method for minimization, Computer Journal, 6 (1963), pp. 163-168.

[12] R. Fletcher And C. M. Reeves, Function minimization by conjugate gradients, Computer Journal, 7 (1964), pp. 149-154.

[13] P. E. Gill And M. W. LeOnARD, Reduced-Hessian quasi-Newton methods for unconstrained optimization, SIAM J. Optim., 12 (2001), pp. 209-237.

[14] P. E. Gill And W. MurRay, Conjugate-gradient methods for large-scale nonlinear optimization, Report SOL 79-15, Department of Operations Research, Stanford University, Stanford, CA, 1979.

[15] P. E. Gill, W. Murray, and M. A. Saunders, SNOPT: An SQP algorithm for large-scale constrained optimization, SIAM J. Optim., 12 (2002), pp. 979-1006.

[16] P. E. Gill, W. Murray, M. A. Saunders, and M. H. Wright, User's guide for NPSOL (Version 4.0): a Fortran package for nonlinear programming, Report SOL 86-2, Department of Operations Research, Stanford University, Stanford, CA, 1986.

[17] P. E. Gill, W. Murray, and M. H. Wright, Practical Optimization, Academic Press, London and New York, 1981.

[18] G. H. Golub And C. F. Van Loan, Matrix Computations, The Johns Hopkins University Press, Baltimore, MD, second ed., 1989.

[19] L. Kaufman, Reduced storage, quasi-Newton trust region approaches to function optimization, SIAM J. Optim., 10 (1999), pp. 56-69.

[20] M. W. Leonard, Reduced Hessian Quasi-Newton Methods for Optimization, PhD thesis, Department of Mathematics, University of California, San Diego, 1995.

[21] D. C. Liu AND J. Nocedal, On the limited memory BFGS method for large scale optimization, Math. Program., 45 (1989), pp. 503-528.

[22] J. L. Morales And J. Nocedal, Automatic preconditioning by limited memory quasi-Newton updating, SIAM J. Optim., 10 (2000), pp. 1079-1096.

[23] J. J. Moré And D. C. Sorensen, Newton's method, in Studies in Mathematics, Volume 24. Studies in Numerical Analysis, Math. Assoc. America, Washington, DC, 1984, pp. 29-82.

[24] J. J. Moré And D. J. Thuente, Line search algorithms with guaranteed sufficient decrease, ACM Trans. Math. Software, 20 (1994), pp. 286-307.

[25] L. NAZARETh, A relationship between the BFGS and conjugate gradient algorithms and its implications for new algorithms, SIAM J. Numer. Anal., 16 (1979), pp. 794-800.

[26] J. NocEdAL, Updating quasi-Newton matrices with limited storage, Math. Comput., 35 (1980), pp. $773-782$.

[27] M. J. D. Powell, Updating conjugate directions by the BFGS formula, Math. Program., 38 (1987), pp. 693-726.

[28] — TOLMIN: A Fortran package for linearly constrained optimization calculations, Report DAMTP/1989/NA2, Department of Applied Mathematics and Theoretical Physics, University of Cambridge, 1989.

[29] M. J. D. Powell And P. L. Toint, On the estimation of sparse Hessian matrices, SIAM J. Numer. Anal., 16 (1979), pp. 1060-1074.

[30] D. F. Shanno, Conjugate-gradient methods with inexact searches, Math. Oper. Res., 3 (1978), pp. 244-256.

[31] D. SIEGEL, Modifying the BFGS update by a new column scaling technique, Report DAMTP/1991/NA5, Department of Applied Mathematics and Theoretical Physics, University of Cambridge, May 1991. 
[32] — Implementing and modifying Broyden class updates for large scale optimization, Report DAMTP/1992/NA12, Department of Applied Mathematics and Theoretical Physics, University of Cambridge, December 1992.

[33] — Modifying the BFGS update by a new column scaling technique, Mathematical Programming, 66 (1994), pp. 45-78. Ser. A.

[34] C. Zhu, R. H. Byrd, P. Lu, And J. Nocedal, Algorithm 7ry8: L-BFGS-B: Fortran subroutines for large-scale bound-constrained optimization, ACM Trans. Math. Software, 23 (1997), pp. 550-560. 